tion of forceps. It seems, however, that many obstetric surgeons, imbued with what may be called tive "furor operativus," make the mere diagnosis of contracted pelvis a reason for the performance of this operation, apart altogether from other considerations. If cases were carefully considered on the lines given above, there is very little doubt that many unnecessary Caesarean sections would be avoided.

If I have gone rather minutely into detail, my excuse is that the more I think over the problem of maternal mortality the more convinced I become that it is only by close attention to every detail, and by co-operation between all the members of the obstetric team, and especially between general practitioners and midwives, that we shall make any real impression upon the number of women who are every year dying preventable deaths.

ReFERENCES

${ }^{1}$ Interim Report of Departmental Committee, para. 117

'Dixon: Pharinicology', 1923, p. 175.

\section{THE ACCESSORY LOBE OF THE AZYGOS VEIN}

BY

H. P. NELSON, M.B., F.R.C.S.

DEMONSTRATOR OF ANATOMY, ST. BARTHOLOMEW'S HOSPITAL AND

GEORGE SIMON, M.B., D.M.R.E.

CHIEF ASSISTANT, X-RAY DEPARTMINT, ST. BARTHOLOMEW'S HOSPITIL

The accessory pulmonary lobe which occurs at the apex of the right lung was first observed by the anatomist in 1778, but its radiographic appearances were not identified until 1923. Since radiographic examination has become the most fruitful method of investigation of the lungs it seems desirable to present a detailed account of the appearances of this infrequent pulmonary variation.

\section{Anatomy}

The accessory lobe of the azygos vein is an additional pulmonary lobe occurring at the right apex, and separated from the remainder of the apex by a fissure containing the vena azygos major. The vena azygos major pursues its usual course behind the oesophagus just to the right of the midline until it reaches the level of the sixth thoracic vertebra, when, instead of arching forwards over the root of the lung, it turns in a lateral direction, dipping into the upper lobe. There, after passing forwards and inclining medially, it terminates in the superior vena cava. The vein, contained in a complete pleural septum, separates the dome of the pleura into two compartments, with the result that an accessory lobe is split off from the medial side of the right upper lobe. This septum consists of both visceral and parietal layers of the pleura, and is so disposed that its outer surface is convex and the lower free margin, which contains between its layers the vena azygos, is arched. It thus forms a kind of meso-azygos (Fig. 1).

The anatomical descriptions of this abnormality are fairly unanimous, and the following account is based on the papers by Geddes, Holtby, and Stibbe; the last reviews all the cases that have appeared in the literature up to 1918. The position of the fissure, and in consequence the size of the accessory lobe, depend on the degree of lateral deviation of the vein. The fissure may split the outer surface of the upper lobe at a point 2 inches below the apex, and the fold will then lie more or less horizontal, the accessory lobe forming the greater part of the apex of the lung. On the other hand, the fissure may merely split off a small tongue-shaped lobe from the mediastinal surface of the lung. The average lies between these two extremes, the fissure usually descending from the apex of the luing. The level of termination of the azygos vein with tire vena cava superior is higher than normal, usually at the junction of the two innominate veins, and meets the lateral instead of the posterior surface of the vena cava. The depth of the fissure varies, but usually approximates to that of a primary lung fissure. In some of the described cases the vena azygos was resting on the eparterial bronchus, while in others there was as much as an inch of lung tissue between the deepest part of the fissure and the bronchus. The bronchus supplying the lobe comes from the eparterial: in three cases it has been described as having an $\mathrm{N}$-shape, first ascending into the lung on the outer side of the fissure and then down under the azygos vein to ascend into the accessory lobe.

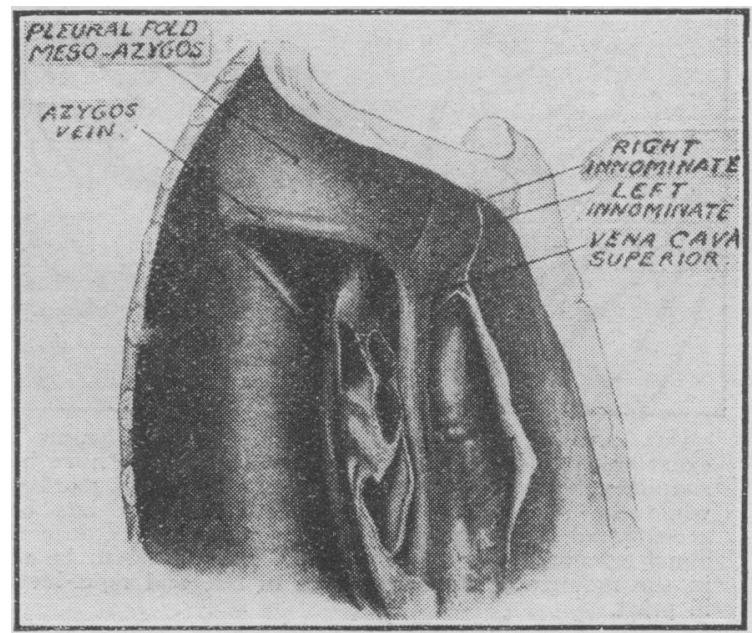

Fig. 1 (after Stibbe).-A case that has been dissected to show the mediastinal surface of the right pleural cavity after removal of the lung and accessory lobe. The pleural fold is shown with the vena azygos major running in its free margin.

In the development of this condition there are two variable factors: the growing lung buds and the growing cardinal veins. The posterior cardinal vein arches forwards across the coelom to join the duct of Cuvier, and it brings with it a fold of primitive pleural membrane, which divides the apex of the pleural-coelomic cavity into a medial and lateral compartment. The lung bud grows upwards and outwards, passing usually lateral to the vein, and with the descent of the heart the vein is drawn downwards and inwards and the medial apex obliterated. Now if there is a slight alteration in the position of either of these factors-a lung bud not lateral enough or the vein too lateral-the pleural fold would impinge against the lung and split off a medial process, which will expand upwards as the accessory lobe. Other accessory lobes, less common than the one here described, have occurred above and below the root of the lung. These other lobes have unfortunately been called " azygos lobes," and it is to distinguish it from these that the lobe we are concerned with has been called " the accessory lobe of the azygos vein." The fissure which separates these " azygos lobes" is lined only with visceral pleura, and is thus similar to a primary lung fissure, but the fissure which separates off the lobe of the azygos vein is lined by both visceral and parietal pleura.

\section{Radiography}

In 1923 Wessler and Jackes first drew attention to a falciform shadow near the right apex occasionally seen in antero-posterior radiograms of the chest. In 1928 Wessler and Bentick observed this shadow in two patients in whom later the opportunity occurred at post-mortem tc compare the anatomy of the lungs with the radiographic appearance. Both of these cases had an accessory lobe of the azygos vein, and they were thus able to prove beyond all doubt the cause of this shadow. 


\section{CASE I}

E. B., a woman aged 35 , was sent for an $x$-ray examination of the chest in order to exclude active tuberculous disease. She had a slight cough, but no abnormal physical signs. The $x$-ray film shows no evidence of disease of the lungs. In the antero-posterior film there is a fine white line which descends from the medial side of the right apex, with a convexity to the right, to end at the level of the second costal cartilage. The distance from the line to the side of the vertebral column

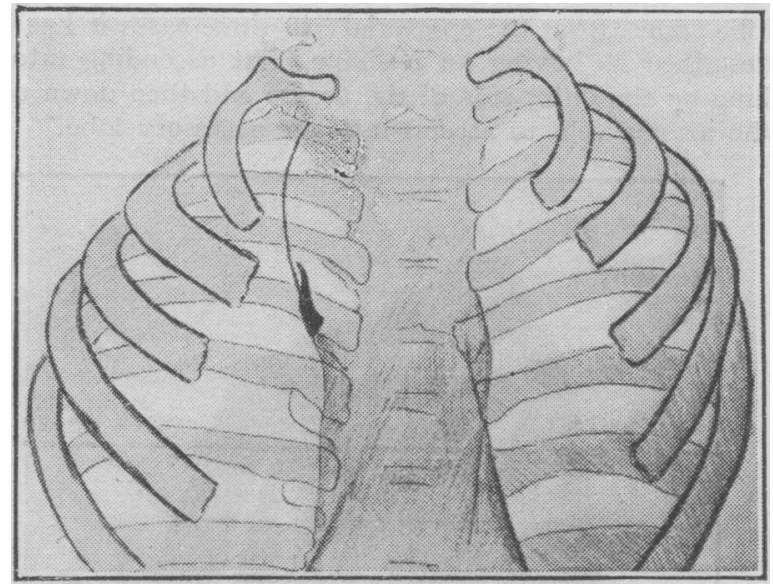

FIG. 2, Case I.-This and the following diagrams are exact tracings of the original radiograms. They have been simplified by leaving out the clavicles and lung markings, while the shadow of the superior vena cava has been accentuated. The radiograms are not shown because this abnormal shadow is not sufficiently noticeable to be scen in the radiograms after reduction in size and reproduction in print.

is $25 \mathrm{~mm}$. at its widest part. The line terminates by running into the side of a horn-shaped shadow, which has an opacity equal to that of the aortic arch. The broad, lower end of this shadow merges into that of the superior vena cava at the level of the upper part of the second intercostal space, while the thin upper end turns medially to lie $1 \mathrm{~mm}$. to the inner side of the line at its lower end. The apex of the lung medial to the line is slightly opaque (Fig. 2).

\section{CASE II}

P. B., a boy aged 15, was admitted to hospital with acute rheumatic pericarditis. An $x$-ray examination of the

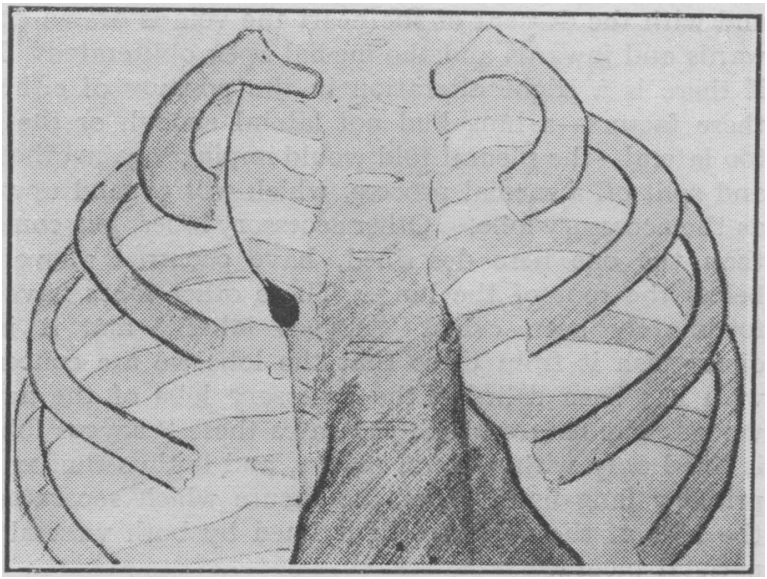

Fig. 3.-Case II.

chest was made, and the antero-posterior film shows a fine white line descending from the right apex, with a convexity to the right, to end at the level of the second intercostal spacc. The distance of the hair line to the vertebral column is $33 \mathrm{~mm}$. The line terminates by running into the apex of a pear-shaped shadow, the broad end of which is continuous with the shadow of the superior vena cava. The great density of this pearshaped shadow makes it stand out very clearly. The apex medial to the line is clear.

\section{CASE III}

D. P., a girl, aged 9, suffering from tuberculous glands in the neck, was sent for $x$-ray examination for the presence of enlarged mediastinal glands. The $x$-ray film shows no evidence of enlarged glands. The antero-posterior film shows a fine white line which descends from the medial side of the right apex, with a convexity to the right, to end at the upper border of the first costal cartilage. The distance of the line from the vertebral column was $15 \mathrm{~mm}$. The line terminates at

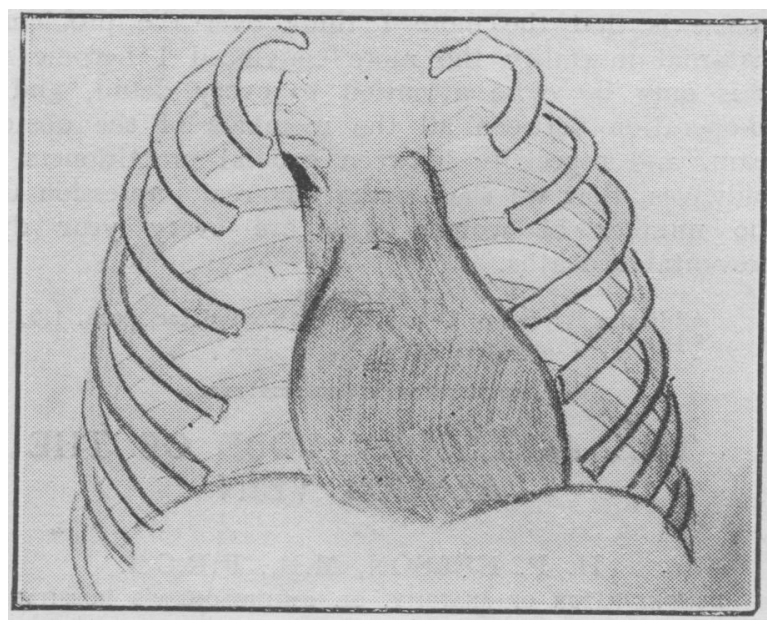

FIG. 4.-Case III.

the upper end of an oblong-shaped shadow, the other end of which is continuous with the superior vena cava. The lung medial to the line is slightly opaque.

The lateral film shows a white'line (considerably wider than that seen in the antero-posterior film) running upwards and forwards from the back of the fourth thoracic vertebra and ending in the first intercostal space on the same plane as the posterior surface of the trachea. This line is 2 inches long.

\section{CASE IV}

F. S, a boy aged 8, suffering from recurrent attacks of bronchitis, was seen at a tuberculosis clinic and sent for $x$-ray examination. The antero-posterior film shows a fino white line which descends from a point at the lateral side of the right apex, with a large convexity to the right, to end at the level of the second intercostal space. The line is $40 \mathrm{~mm}$. from the vertebral column at its widest part. The shadow at the lower end of the line is quite different from that of the threc

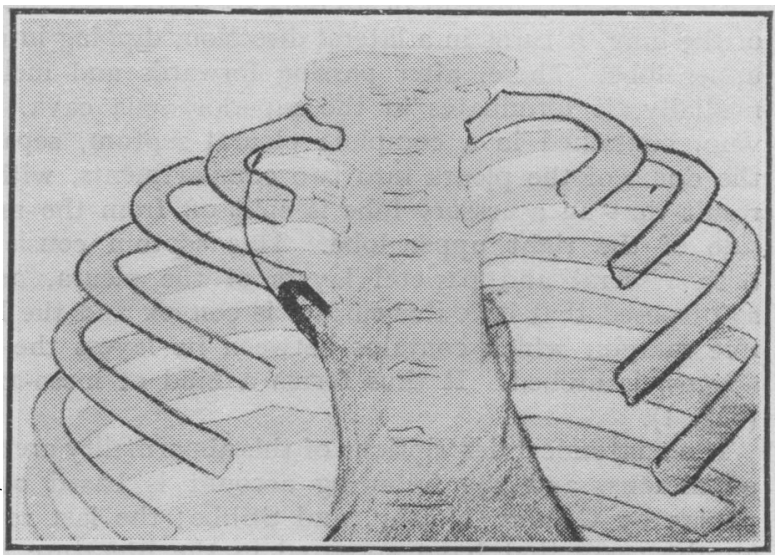

FIG. 5.-Cast IV.

preceding cases. There is a horseshoe-shaped shadow with the concavity pointing downwards and inwards, the upper limb is on the level of the second costal cartilage, and the lower limb in the second intercostal space. The line can be seen to run into the outer side of the lower limb of the horseshoe-shadow. This shadow is only slightly denser than that of the superior vena cava.

The normal lung fissures can only be seen in radiograms of the chest if the incidence of the rays is in line with the 
plane of the fissure. Thus the septum which separates the accessory lobe only casts a shadow for that part of its course which is in line with the rays, when it appears as a linear shadow. The position of the line will vary with the width of the accessory lobe. The topography of the vena azygos, which can be seen at the lower end on the medial side of the linear shadow, exhibits considerable variation. This variation depends on the depth of the fissure. In Case IV the whole of the arch can be seen projecting well out into the lung field; in Case II the vein is lying close against the side of the mediastinum, and the arch is coming almost directly forwards, which accounts for its great density as compared with Case IV. In the other two cases the vein is intermediate in position and density of shadow between that of Cases II and IV. Rawling states that the normal vena azygos opens into the superior vena cava at the level of the lower part of the second right intercostal space: the level of termination is seen in these cases to be higher than in the normal, Cases II and III being opposite the first interspace and Cases I and IV opposite the second costal cartilage.

\section{Pathology}

The only pathological condition that has been found in this lobe is due to obstruction of its bronchus by the vena azygos. Muller described the case of a boy, aged 18, who had myeloid leukaemia. The radiogram of the chest showed an abnormal shadow projecting laterally from the mediastinum above the hilum of, the right lung, which was thought to be due to enlarged lymphatic glands. The boy died of the leukaemia, and the post-mortem revealed that the shadow seen in the radiogram was due to an accessory lobe of the azygos vein, which showed important pathological changes. The bronchus leading to the lobe was narrowed to a slit at its point of contact with the vein, while beyond this the bronchus opened up into a series of bronchiectatic sacculi filled with mucus. Muller's case suggests that the opacity observed in the lobe in two of our cases might be due to a partial obstruction of the bronchus. Bentick and Wessler observed the shadow of an accessory lobe in a patient who had a carcinoma of the right lower lobe. A pleural effusion developed, and at the same time the hair line became progressively wider, probably owing to an interlobar effusion.

Apart from these two cases there is no record of any pathological state associated with this congenital abnormality.

\section{Frequency}

In 1916 Stibbe reviewed the twenty-three cases that had been reported in the literature since 1778, when Wrisberg first described the condition. Nineteen of these cases were reported in anatomical journals between 1866 and 1899 .

More accurate statistics can now be obtained from the radiological departments of large chest hospitals. Mather, from the Royal. Chest Hospital, Liverpool, reports four cases in 3,000 consecutive radiograms of the chest; while Sparks (personal communication), at the City of London Hospital, Victoria Park, has had six cases in 6,000 consecutive radiograms of the chest. Thus the incidence of this abnormality is approximately 1 per 1,000 of the population. The condition is also much more frequent in males than females.

\section{BibliogRaphy}

$$
\text { Anatomy }
$$

Wrisberg, H. A. Göttingen, 1777, viii, 14.

Dévé, M.: Bull. de la Soc. Anat. de Paris, 1899, lxxiv, 489.

Gedrles, A.: Journ. Anat. and Phy's., 1910, xiv.

Holtby: Ibid., 1915, xlix.

Stiblye, E. P.: Journal of Anatomy, 1918, iii, 305.

\section{Radiology}

Wessler and Jackes: Clinical Roentgenology of Diseases of the Chest, p. 17. 1923. The Southworth Company, Troy, N.Y. Velde, G.: Fortschr. a. d. Geb. de Roentgenstrahlem, 1927, xxxvi, 315.

Hjelm and Hulten: Acta Radiol., April, 1928, ix, 126.

Bentick and Wessler: Amer. Journ. Roent., 1928, xx, 1.

Mather and Coope: Brit. Journ. Radiol., 1928, 481.

Stoloff: Amer. Journ. Rocut., 1929, xx, 5. ,

\section{THE KNEE-JERK AND ITS VARIATION AN INDICATION FOR SYMPATHETIC RAMISEC'TION OR TRUNK SEC'TION}

\author{
BY \\ N. D. ROYLE, M.D., CH.M., F.C.S.A. \\ SYDNEY
}

There are a number of diseases in which the deep reflexes, such as the knee-jerk, are increased in excitability and have a prolonged relaxation time, with few or no oscillations when coming to rest after elicitation. This occurs in Raynaud's disease, some cases of Buerger's disease, Hirschsprung's disease, and in certain other vasospastic conditions. Dr. Harry Harris has found similar phenomena exhibited by patients suffering from certain types of renal pain. These diseases are due to abnormal activity of the sympathetic nervous system, as is distinctly indicated by the manner in which each condition responds to operations on this system. The presence of these abnormal reflex phenomena may be used as an indication for operation, because they disappear, or are modified, after the interruption of sympathetic influence.

\section{How to Test the Knee-jerk}

The knee-jerk is a myotatic reflex, and depends on the stretching of the quadriceps for its elicitation. This is usually accomplished by flexing the knee when the patient is reclining, or sitting upright in a chair. The first position does not adequately stretch the quadriceps to induce tone. The second position allows the effect described by Magnus and de Kleijn to come into playthat is, an increase in tone in all extensor groups of muscles caused by contraction of the extensor muscles of the head, thus exaggerating the amount of tone present in the tested muscles.

\section{Reinforcements of the Knee-jerk}

Various methods of reinforcing the knee-jerk are used, such as pressing the hands together and making the patient look at the ceiling, but these reinforcements interfere with the exact state of the knee-jerk, in that they call into action a cortical contribution to tone.

\section{The Ideal Position for Testing a Knee-jerk}

To test a knee-jerk the patient should be in a reclining position. The head and back should be supported, so that the contraction of extending muscles of the neck is not evoked. The knee should be bent and allowed to swing freely over the edge of the bed or couch. In this position the quadriceps muscle is stretched, and this stretching induces whatever tone the quadriceps is capable of exhibiting.

\section{Phases in the Knee-jerk}

When the patellar tendon is tapped there is a phasic response in which the knee extends, and a subsequent variable phase. If tone is exaggerated the leg will stay. 